\title{
Noise methods in pattern perception
}

This experiment investigated 4 methods of inducing visual noise at 7 levels of noise. Forty-six $S$ s were presented 4800 sets of 3 pattems, with the task of judging which of 2 noisy pattems was more similar to the prototype patter. The noisy pattems were generated by adding, moving, deleting or both adding and deleting elements of the prototype. All possible comparisons of each noise method were made at each noise level and the results plotted. The results show that the variaus methods of introducing noise do not produce identical results, and that the similarity assessment is dependent on noise level. Explanations are offered to account for the results.

Visual noise is a term to describe the variation of a visual signal due to changes in the elements comprising the signal and/or its surround. This noise can have many levels, i.e., any number of eleménts might be manipulated and, in addition, can take several forms. The purpose of this investigation was to examine several forms and levels of visual noise as they have been used in pattern perception studies.

Typically in investigations using noise patterns a prototype or master pattern is constructed and the noise patterns are generated by performing some type of manipulations on this prototype. French (1954) simply added elements to the prototype and labeled them noise patterns. Hillix (1960) moved elements from one location to another. Arnoult and Price (1961) both added and deleted elements in a number of prototypes to make noise patterns. Fitts and Leonard (1957) produced noise by manipulating the information content of patterns. In the final analysis all their methods involved either adding, deleting, or moving edements in the patterns or some combination of these.

The question naturally arises as to whether these methods of producing noise can be equated psychologically. If one assumes that it is not possible to equate them, the question then becomes how do they differ. Arnoult and Price (1961) compared their data with that of Hillix (1960) and concluded that some of the differences found might be attributed to the two different methods employed to produce the noise patterns.

An examination of the methods used to make noise patterns points to some factors that should be expected to lead to behavioral differences. For the method in which elements of the signal are moved from one location to another to produce noise, it is obvious that for every element moved two portions of the pattern are changed, the portion from which the element was taken and the portion in which it is placed. Adding or deleting elements, however, involves only one portion of the pattern. If $n$ elements in a pattern are added or deleted, then, this will produce only half as much configurational difference as moving n elements. In the moving of elements method, however, the number of elements composing the signal remains the same which, of course, is not true when elements are added or deleted.

The method of producing noise employed by Amoult and Price (1961) demands other considerations. For purposes of explanation assume that filled cells of a matrix constitute the signal. Here $n$ cells are selected at random to be changed. If the cell chosen is filled, it is changed to the unfilled state, but if unfilled it is changed to the filled state. Elements of the signal pattern, then, are both added and deleted. The probability of only adding or only deleting cells with this method is contingent upon the ratio of filled to unfilled cells in the prototype. If this ratio were close to 1.0 as you would expect in the case of Arnoult and Price (1961), (prototypes were constructed by filling cells in a matrix with $p=.50$ ), and if only a few elements were to be changed, the probability of only adding or deleting cells would be higher than if a large number of elements were to be changed. This method is identical to the moving elements method on those occasions when equal numbers of cells are added and deleted.

To assess some of the relationships between noisy visual signals and to determine how different methods of producing noise might be judged with respect to similarity, a study was conducted in which various methods of noise generation were compared with each other.

\section{Apparatus}

\section{METHOD}

Patterns were presented to Ss via closed-circuit television. Three separate patterns appeared on the screen at one time. Each of these three patterns appeared as a group of illuminated hexagonal cells and were, in fact, generated by lighting chosen elements of separate groups of hexagonal lucite rods. The rods were $1 / 2 \mathrm{in}$. in diameter and $2 \mathrm{in.} \mathrm{long.}$ They were bonded tightly together in rows and columns of eight. When viewed with the rods end on, it appeared honeycomb in texture. A hole drilled in one end of each rod accommodated an 8-V lamp. Each rod was electroplated with a translucent material such that when a lamp was lit it illuminated the rod at the opposite end, but there was no noticeable spill over of light onto adjacent cells. Three such matrices were constructed with independent control of each cell in each 
matrix. A paper tape reader was used to designate the particular cells to be illuminated on any trial. The patterns of light were picked up by a TV camera and relayed to TV monitors located in three subject booths. The apparatus was capable of presenting a different set of three patterns every $5 \mathrm{sec}$.

\section{Stimuli}

The stimuli consisted of 4800 sets of three patterns. Each set of patterns contained a randomly generated prototype pattern and two test patterns. The prototype patterns were constructed by lighting empty cells with a probability of .50; the lit cells constituted the signal. The test patterns were variations of the particular prototype with which they were paired. There were four methods of introducing noise: (1) lit cells were added to the prototype; (2) lit cells were deleted; (3) lit cells were "moved," i.e., a lit cell would be turned off at one location and at another location a cell would be lit; and (4) the state of randomly chosen cells was changed, i.e., cells were chosen at random to be changed and if the chosen cell was lit it was changed to an unlit cell, but if unlit changed to a lit cell. This was called add-delete. In instances where it applied, a rule was imposed that no cell could be changed more than once.

The level of noise was determined by the number of cells manipulated. This ranged from 0 to 28 percent, in steps of about 4 percent of the total possible cells, 64 . This involved $0,3,5,8,10,13,15$, or 18 cells when the values were rounded off to the nearest whole number. The move method of generating noise considered only the number of cells "moved" in calculating the noise level and not the number changed.

The 4800 sets of patterns were divided into 10 sets of 480 . Within each group of 480 the four methods of noise generation were paired equally often. Each level of noise was presented an equal number of times with each noise method pairing. While the noise level changed between sets of patterns in a random sequence, within any one set of patterns the test stimuli were always at the same level of noise.

\section{Subjects}

The Ss were 26 male college students and 20 housewives. They were obtained from a subject pool maintained by the laboratory for psychophysical research and were paid by the hour.

\section{Procedure}

The Ss were tested in groups of three or fewer. Each day for 10 days they responded to a different group of 480 patterns. Four separate 10-day periods were necessary to run all Ss. Testing time was approximately $50 \mathrm{~min}$ per day. The Ss were instructed to indicate by pressing a switch which of the two test stimuli, located below and on either side of the prototype, was more similar to the top pattern. All Ss were allowed to view each set of patterns as long as they desired, but were told to proceed as rapidly as possible. The test stimulus judged more similar to the prototype was presumed to have been less distorted by the method used to produce noise.

\section{RESULTS AND DISCUSSION}

Each $S$ made a total of 100 responses to each combination of noise methods at each level of noise. For each method-level combination the proportion of times that each noise method was judged more similar was obtained. These data are plotted in Fig. 1. The amount of noise is indicated on the abscissa in percentage values. The dashed line indicates the response proportion at which no consistent preference is indicated for either type of noisy figure; thus it might be called the point of subjective equality. The solid black line connects the mean scores for all Ss at each level of degradation. The vertical lines at each data point indicate the range of one standard deviation about the mean. A control condition (not plotted on these graphs) presented three identical patterns; i.e., the noise level for both test patterns was equal to zero. Judgments on these trials did not deviate significantly from chance.

Consider first the comparisons between the move method of noise and all other methods ( $A, E$, and $F$ of Fig. 1). As described earlier the move method of producing noisy patterns involves manipulating twice the number of total pattern elements. It would be reasonable to assume, then, that a higher probability exists for choosing a pattern other than a move pattern. This is indeed the case in Fig. $1 \mathrm{~F}$ and at the lower noise levels of Figs. 1A and 1E. However, in Fig. 1E the move patterns actually have a higher probability of being judged more similar at the high noise levels. In Fig. $1 \mathrm{~A}$ the proportion of responses to the move patterns also increases

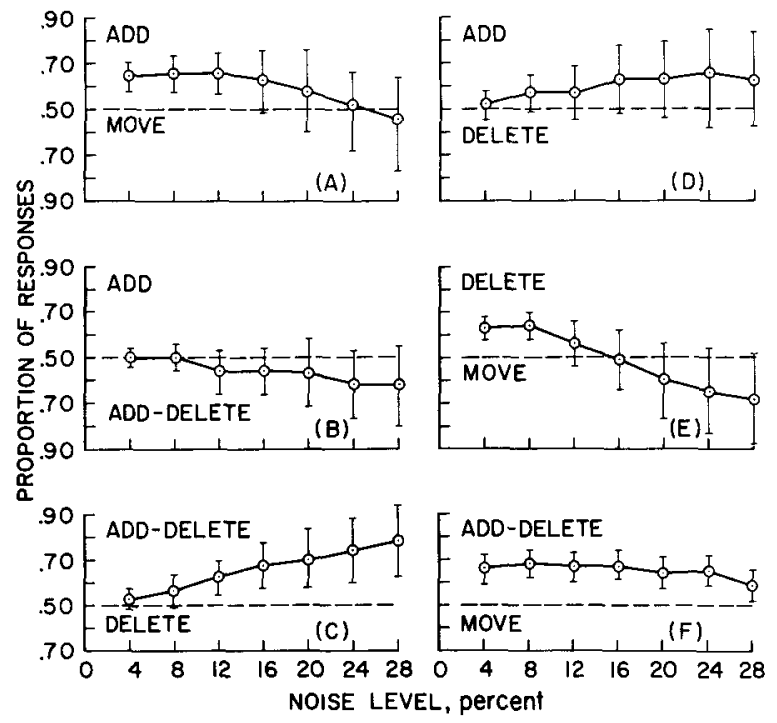

Fig. 1. The proportion of responses to each type noise pattem when compared with all types of noise at each noise level. 
at the higher noise levels, though not as dramatically as in Fig. $1 \mathrm{E}$.

One possible explanation for the trends of the curves found in $A$ and $E$ of Fig. 1 takes into account the ratio of lit to unlit cells between the noisy patterns and the prototypes. This will be referred to as the ratio criterion. The assumption is that on any trial the noisy pattern with a lit-to-unlit ratio closest to that of the prototype has the higher probability of being judged more similar to the prototype. The add and delete noisy patterns in all cases experience an increasing change in this lit-to-unlit ratio as the noise level is increased. However, this ratio remains identical between the move noisy patterns and the prototypes regardless of noise level. If this ratio is a criterion, then, it would be expected that as the noise level increases in Figs. $1 \mathrm{~A}$ and $1 \mathrm{E}$ the ratio criterion would become more powerful and thus offer more competition as a response determinant.

The curve in Fig. $1 \mathrm{~F}$ is also in accordance with a ratio criterion. The add-delete noise method predicts that (statistically) equal numbers of cells will be added and deleted. This means, of course, that the ratio of lit-to-unlit elements remains close to that of the prototype at all noise levels. As indicated previously, the ratio remains constant for the move method. In Fig. $1 F$ the preference is for the add-delete patterns at all noise levels but it is important to note that this curve has no slope. The explanation offered here is that while changing twice the number of elements in the move method decreases the probability of responding to this type of noisy pattern, the ratio criterion operates to keep the judged similarities about the same except for this difference.

Agreement with the ratio criterion is also reflected in graphs $\mathrm{B}$ and $\mathrm{C}$ of Fig. 1 where add noisy patterns or delete noisy patterns are paired with the add-delete noisy patterns. The add-delete patterns acquire a greater proportion of the responses as the noise level increases. Here again the ratio changes proportionate to the noise level for the add and delete patterns while it remains essentially the same for the add delete patterns.

The pairing of add noisy patterns with delete noisy patterns (Fig. 1D) is the only pairing in which the ratio criterion does not operate. In this case the trend is for add patterns to be judged more similar as nolse level increases. This is probably related to the fact that in add patterns the original configuration of the prototype is always present despite any increase in noise level, whereas, of course, each element deleted subtracts from the prototype configuration. This conclusion is supported also when Figs. 1A and $1 \mathrm{E}$ are compared as well as Figs. 1B and 1C. The add pattern curves are much slower and less dramatic in their change as a function of noise level than are the delete patterns when paired with move and adddelete patterns.

One rather striking aspect of the data is seen when the standard deviations in Fig. $1 \mathrm{~F}$ are compared with those of the other graphs. In all other cases there is a steady increase in the standard deviations as a function of increased noise level, whereas in Fig. $1 \mathrm{~F}$ the standard deviations are about equal at all noise levels. The explanation for this effect probably lies in the similarity of the noise methods for add-delete and move patterns, but further research will be required to examine this effect thoroughly.

The primary purpose of this experiment was to indicate how various methods of generating noise patterns that have been reported in the literature might differentially affect the perception of patterns. It is clear that behaviorally these several methods investigated are not identical in effect. Other factors such as pattern size, the separation of elements in the patterns, different means of display, etc., would probably lead to different trends. These results, nevertheless, clearly demonstrate that the kind of noise that is present is an important factor in the perception of patterns.

\section{References}

Arnoult, M. D., \& Price, C. W. Pattern matching in the presence of visual noise, J. exp. Psychol., 1961, 62, 372-376.

Fitts, P. M., \& Leonard, J. A. Stimulus correlates of visual pattern recognition-a probability approach. Final Report of Office of Naval Research, Contract Nonr 495(02), 1957.

French, R. S. Pattern recognition in the presence of visual noise. J. exp. Psychol., 1954, 47, 27-31.

Hillix, w. A. Visual pattern identification as a function of fill and distortion. J. exp. Psychol., 1960, 59, 192-197.

(Accepted for publication April 18, 1967.) 